Tecno Lógicas

ISSN 0123-7799

Vol. 17 , No. 32 , pp. 67-74

Enero-junio de 2014
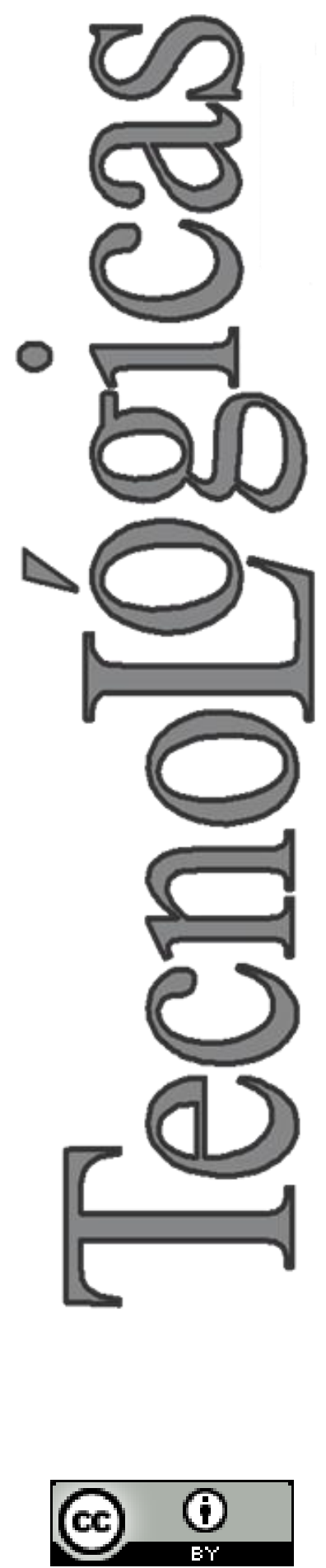

\section{Plataforma virtual para el mando local y remoto de un brazo robótico de apoyo para la educación en ingeniería}

\section{Virtual environment for local and remote control of a robot arm for support in engineering teaching}

\author{
José T. Buitrago-Molina ${ }^{1}$, \\ Juan S. Carvajal-Guerrero ${ }^{2}$, Camilo A. Zapata-Castillo ${ }^{3}$
}

Recibido: 15 de noviembre de 2013 , Aceptado: 18 de diciembre de 2013

Como citar / How to cite

J. T. Buitrago-Molina, J. S. Carvajal-Guerrero y C. A. Zapata-Castillo, "Plataforma virtual para el mando local y remoto de un brazo robótico de apoyo para la educación en ingeniería", Tecno Lógicas, vol. 17, no. 32, pp. $67-74,2014$.

1 Magister en Automática, Escuela de Ingeniería Eléctrica y Electrónica, Universidad del Valle, Cali-Colombia, jose.buitrago@correounivalle.edu.co

2 Ingeniero Electrónico, Escuela de Ingeniería Eléctrica y Electrónica, Universidad del Valle, Cali-Colombia, juancarvajal16@gmail.com

3 Ingeniero Electrónico, Escuela de Ingeniería Eléctrica y Electrónica, Universidad del Valle, Cali-Colombia, camiloa124@hotmail.com 


\title{
Resumen
}

En este artículo se presenta el diseño e implementación de una plataforma virtual, que permite la simulación y mando local y remoto del brazo robot tipo SCARA llamado UV-CERMA, presente en el Laboratorio de Robótica de la Escuela de Ingeniería Eléctrica y Electrónica de la Facultad de Ingeniería de la Universidad del Valle, el robot UVCERMA ha estado subutilizado desde hace algunos años debido a lo obsoleto de su sistema de control. La plataforma, enfocada con los lineamientos de educación en ingeniería está compuesta por dos aplicaciones que permiten la simulación y el mando y control locales y remotos para el robot, una de las aplicaciones se programó haciendo uso del paquete de National Instruments LabVIEW y la otra aplicación se realizó por medio de software libre, por medio del lenguaje de código abierto Java, ambos desarrollos implementan los modelos cinemático directo y cinemático inverso, un módulo para la planificación y ejecución de trayectorias, otro módulo para el monitoreo de variables y un modelo 3D del robot. Para la manipulación del robot se tiene una interfaz con un joystick, que lo hace más versátil. Las aplicaciones se comunican al robot real mediante una tarjeta de adquisición de datos de National Instruments NI USB-6211, y para el mando remoto la plataforma cuenta con una arquitectura cliente/servidor usando sockets TCP/IP.

\section{Palabras clave}

Interfaz, plataforma virtual, brazo robot, Java, LabVIEW.

\begin{abstract}
This paper presents the design and implementation of a virtual platform that allows simulation and local and remote command and control of the SCARA robot arm called UV-CERMA, which is installed at the Robotics Laboratory of the Escuela de Ingenieria Eléctrica y Electrónica, Facultad de Ingeniería, Universidad del Valle. The robot has been underutilized for some years due to the obsolete control system. The platform, focused on the engineering education methodology, consists of two applications which simulate the robot and permit its remote and local command and control. One of the applications was implemented on LabVIEW software of National Instruments and the other application was programmed on free software using the open source language Java. Both applications implement forward kinematics and inverse kinematics, have a module for trajectories planning and operation, a module for monitoring the values of the variables and a three dimensional model of the robot. To manipulate the robot, an interface with a joystick was developed, enhancing the versatility of the platform. The applications communicate with the real robot using the National Instruments data acquisition card NI USB-6211, and for the remote connection they have a client-server architecture using TCP/IP sockets.
\end{abstract}

\section{Keywords}

Interface, Java, LabVIEW, robot arm, virtual platform. 


\section{INTRODUCCIÓN}

Actualmente, la formación experimental de los estudiantes de ingeniería se está convirtiendo en la llave que abre las puertas a la prosperidad dentro del campo industrial, dicha formación es generalmente de carácter presencial, sin embargo, debido a diversos factores económicos, espaciales y temporales, este método de enseñanza no es suficiente a veces para ofrecer una calidad y cobertura adecuadas en ciertas materias de carácter técnico [1], [2].

Con la llegada de las tecnologías telemáticas y de Internet (TIC's), aparece una nueva era de conectividad entre máquinas que proporciona formas innovadoras para adquirir, analizar y presentar la información. Este hecho ha abierto un mar de posibilidades nuevas para la formación a distancia, por esta razón, muchos de los grupos de investigación de carácter técnico, trabajan en el desarrollo de laboratorios virtuales y remotos para la docencia en ingeniería [2], [3].

Dentro del campo de la robótica, se han desarrollado varios laboratorios virtuales y remotos. Su funcionalidad se basa en el control y teleoperación a distancia tanto de robots móviles como industriales. Estas aplicaciones permiten al estudiante adquirir rápidamente conceptos y habilidades para controlar un robot, comprender su funcionamiento $\mathrm{y}$ estar preparado para manipularlo adecuadamente.

Con el propósito de ampliar la cobertura en la formación académica y de investigación y de optimizar los escasos y costosos recursos de los que se disponen en robótica industrial, se presenta el desarrollo de este proyecto con la intención de aplicar las metodologías de aprendizaje activo para la educación en ingenierías.

El proyecto consistió en concebir e implementar una herramienta de software, que permite la simulación y mando del brazo robot tipo SCARA (Selective Compliance Assembly Robot Arm) llamado UVCERMA presente en el Laboratorio de
Robótica de la Escuela de Ingeniería Eléctrica y Electrónica, el cual ha estado subutilizado desde hace algunos años debido a lo obsoleto del sistema de control.

\section{LABORATORIOS VIRTUALES Y REMOTOS}

Después de realizar una lectura reflexiva de algunas definiciones propuestas en algunos trabajos previos [4]-[6], se acogieron las presentadas en [7] como se presentan a continuación:

Laboratorio Virtual (LV): Instrumento simulado contenido en uno o más computadores, conectados o no entre sí, con capacidades de gestión y/o aprendizaje de contenido.

Laboratorio Remoto (LR): Lugar o entorno cuya función es realizar un control sobre un sistema físico a distancia, con el objetivo de teleoperar un sistema real, realizar experimentos y acceder a los datos a través de la red para obtener medidas.

Laboratorio Virtual y Remoto (LVR): sistema físico real y/o simulado accesible desde Internet con capacidades de gestión, aprendizaje de contenido y/o reservas de recursos compartidos.

Existen muchas clasificaciones sobre ambientes de aprendizaje, recursos locales reales, recursos locales simulados, los recursos remotos simulados y los recursos remotos reales, donde se han caracterizado herramientas tecnológicas de apoyo a la educación en control [8]. Aplicando conceptos como estos se han desarrollado laboratorios tanto virtuales como remotos, en donde se muestran resultados en tipos diferentes de robots industriales como robots de configuraciones SCARA o articular con interfaz gráfica en Java, comandados por teclado o botoneras y enfocados al entrenamiento de usuarios, analizando su impacto en el aprendizaje [9]-[13] y otros implementan la interfaz con LabView y algoritmos inteligentes para un robot en aplicación médica [14] y se hacen algorit- 
mos de control para planificar trayectorias por medio de comandos verbales [15] o siguiendo un patrón de luz [16].

Según las definiciones anteriormente planteadas se presenta en este artículo una plataforma que se incluye en la clasificación de Laboratorio Virtual y Remoto, LVR.

\section{DESCRIPCIÓN DE LA PLATAFORMA}

\subsection{Infraestructura del sistema robótico}

En general, un sistema robótico lo constituyen los elementos físicos que lo soportan y el ambiente tecnológico sobre el cual actúa el operador, como se presenta en la Fig. 1.

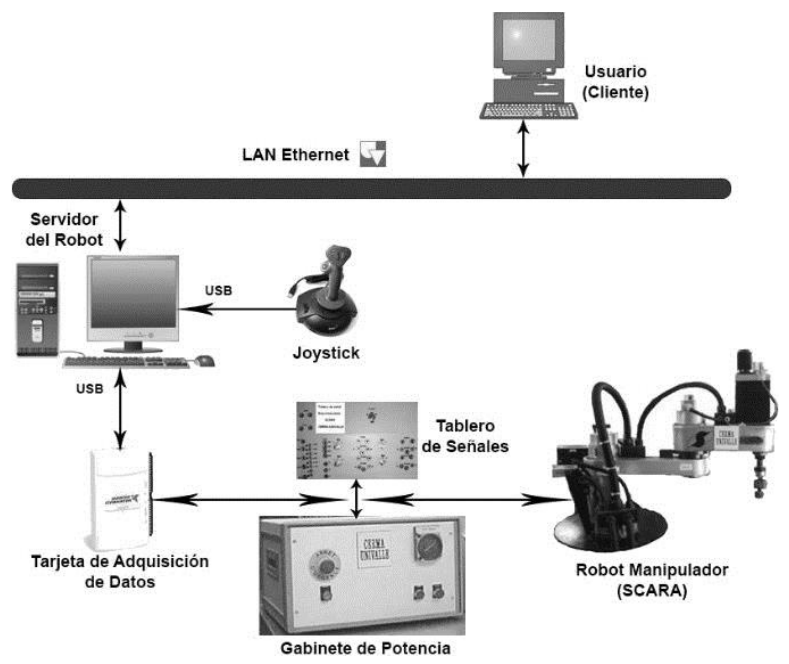

Fig. 1. Esquema del Sistema Robótico. Fuente: Autores

El esquema presenta los subconjuntos funcionales y su interconexión, conformando una estructura de cooperación permanente, concebida como un sistema modular, con el fin de posibilitar la actualización tecnológica a medida que se estudie, investigue y desarrolle cada una de sus partes. Los cuatro elementos principales que constituyen dicha estructura son el soporte informático, el sistema de adquisición de datos, el sistema electrónico de potencia y la estructura mecánica.

Para el soporte informático se dispone de un computador en el interior del laboratorio que hace las veces de servidor del robot y se encuentra conectado a la red institucional de la universidad. Este computador se comunica por USB con los dispositivos de adquisición de datos, que son las tarjetas NI USB 6211 y NI USB 6009. El sistema de potencia lo conforman los variadores de velocidad para los motores presentes dentro de la estructura mecánica del robot y las fuentes de alimentación con sus respectivos interruptores manuales y protecciones.

La configuración física del Robot UVCerma es de tipo SCARA (Selective Compliance Assembly Robot Arm), como se muestra en la Fig. 2. Los enlaces, o articulaciones motorizadas, están en configuración $R R P R$, o sea: dos articulaciones rotativas, para mover el Robot en el plano de dos dimensiones; una articulación prismática, que extiende el movimiento a una tercera dimensión; y una última articulación rotativa, asociada al elemento terminal completando la operación con giros sobre el plano. El elemento terminal, sobre el cuarto eje, soporta un electroimán que permite recoger y colocar objetos ferromagnéticos.

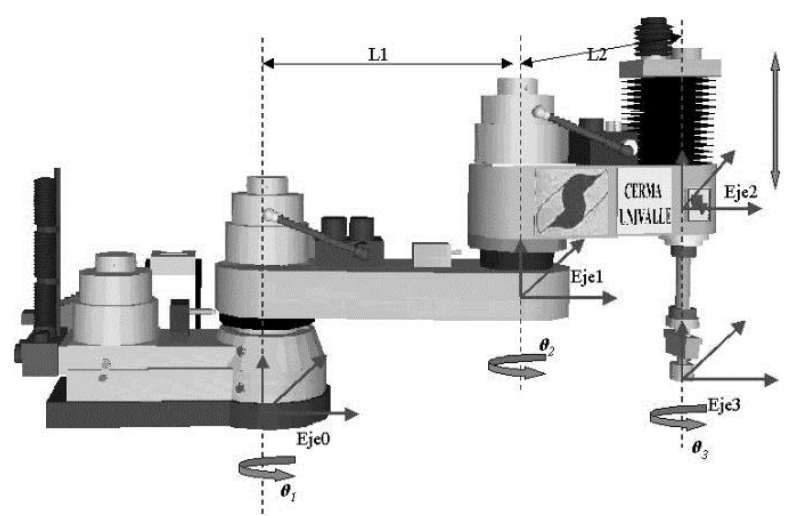

Fig. 2. Esqueleto Funcional del Manipulador. Fuente: Autores 
J. T. Buitrago-Molina et al. / Plataforma virtual para el mando local y remoto de un brazo robótico de apoyo para la educación en ingeniería

\subsection{Arquitectura de la plataforma virtual}

La arquitectura de la plataforma se presenta en el esquema general presentado en la Fig. 3.

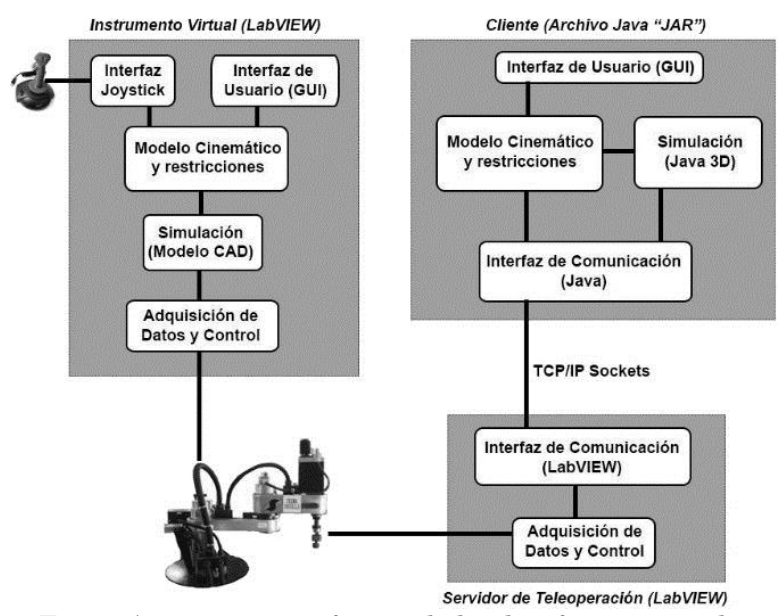

Fig. 3. Arquitectura software de la plataforma virtual y remota. Fuente: Autores

Como se puede observar, la plataforma está compuesta por diferentes componentes software dentro de los cuales se encuentra un instrumento virtual desarrollado en LabVIEW presente en el computador servidor del laboratorio, este cuenta con una interfaz gráfica de usuario, un módulo de comunicación para un dispositivo joystick, y el modelo cinemático que se comunica tanto con una versión simulada en $3 \mathrm{D}$ del Robot cuyas partes fueron modeladas en el software Autodesk Inventor, como con el módulo para la manipulación del robot real.

Por el lado del cliente se tiene una aplicación en Java para el acceso remoto, la cual también cuenta con el modelo cinemático del robot dentro de su núcleo y un modelo en 3D del brazo robótico diseñado por medio de java 3D. Esta aplicación cliente incluye un módulo que se encarga de la comunicación vía TCP/IP con el instrumento virtual servidor que intermedia en la comunicación con el robot real.

\section{ANÁLISIS Y DISCUSIÓN DE RESULTADOS}

Un primer producto de este proyecto es la interfaz Hombre-Máquina implementada en LabVIEW (ver Fig. 4).

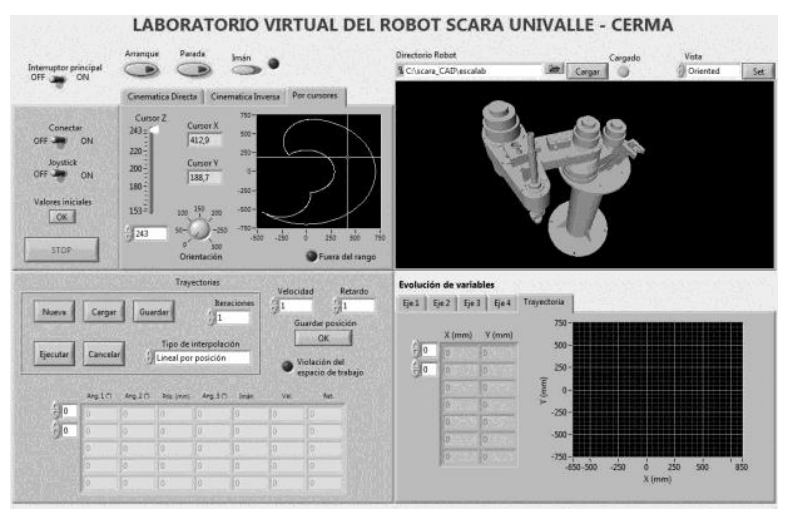

Fig. 4. Interfaz gráfica de usuario LabVIEW. Fuente: Autores

Dicha interfaz contiene las siguientes funcionalidades: La capacidad de navegar en el mundo virtual donde se muestra el modelo en 3D del brazo robótico y que corresponde a la principal realimentación visual que tiene el usuario. Un panel de control de posición separado en pestañas, donde la primera pestaña corresponde al control de las articulaciones, la segunda al control del elemento terminal del robot y la tercera pestaña corresponde al control por cursores. En la parte inferior de la interfaz de LabVIEW se encuentra la funcionalidad para la construcción de trayectorias punto a punto, con las opciones de ejecutar, guardar y cargar los puntos programados, además de un panel para monitorear las variables al tiempo que se va ejecutando la trayectoria.

Otro producto del proyecto es la interfaz gráfica de usuario desarrollada sobre Java, la cual es un archivo ejecutable (JAR) que se encuentra disponible para los usuarios que deseen hacer uso de ella desde cualquier computador, en el caso de que 
se requiera la teleoperación del sistema es necesario ejecutarla en un computador conectado a la red institucional de la Universidad. Dicha interfaz se presenta en la Fig. 5.

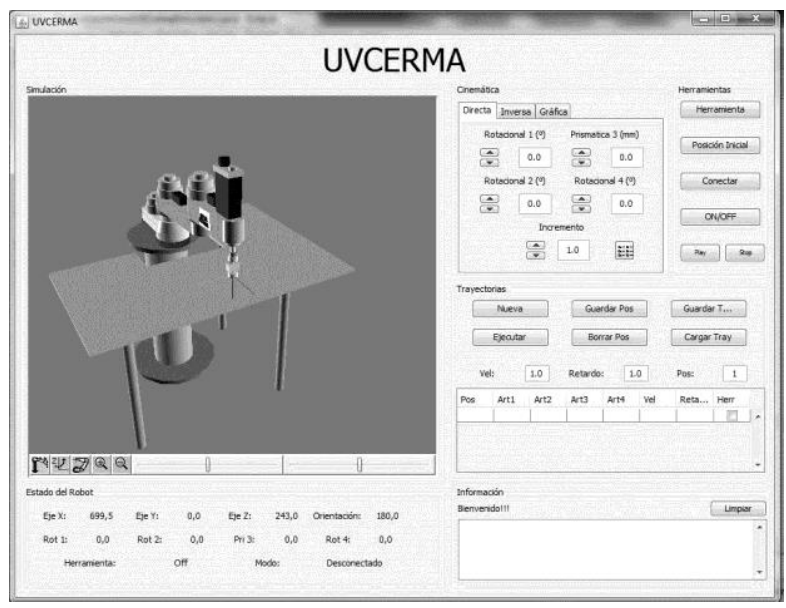

Fig. 5. Interfaz gráfica de usuario JAVA. Fuente: Autores

Esta interfaz se encuentra divida en paneles, uno de los cuales es el encargado de presentar el modelo 3D del Robot brindando al usuario una realimentación visual del estado actual del Robot, ya sea en simulación o en teleoperación. Otro panel está relacionado al control de posición, el cual cuenta con tres pestañas, una para el mando mediante cinemática directa, otra para la cinemática inversa y una última para un control gráfico. Otro de los paneles brinda la posibilidad de crear, ejecutar, guardar o cargar trayectorias, definidas por una serie de pasos obligados. Un panel para el aviso de los eventos de la interfaz, otro para el estado del robot y un último para herramientas varias entre las que se destaca la opción para conectarse con la aplicación servidora para el modo de teleoperación.

El programa servidor es una aplicación desarrollada en LabVIEW que se encarga de comunicarse bidireccionalmente con la aplicación cliente de java, este servidor cuenta con dos grandes módulos. Un módulo configura el socket TCP/IP para la comunicación, fragmenta la trama recibida y arma la trama que se envía con los datos medidos reales, mientras que el otro modulo se encarga del control de posición y la comunicación con la tarjeta.

Uno de los impactos principales de la plataforma es el apoyo en formación de los nuevos profesionales que requiere el país en el campo de la ingeniería, con competencias en robótica industrial que es una tecnología que se transfiere a los sectores productivos. Para el logro de este proyecto, se utilizaron herramientas, métodos $\mathrm{y}$ técnicas informáticas de uso en entornos industriales (caso LabVIEW), esto es consecuente con la formación en ingeniería en el medio. De esta forma se hace un aporte en el sector académico, como parte esencial de las actividades de investigación y desarrollo (I+D).

La plataforma de experimentación en robótica industrial será de mucha utilidad para obtener conocimientos y hacer investigación y entrenamiento (inclusive al sector productivo) en esta temática. Con este ambiente se abarcan los contenidos de un curso de robótica industrial (volumen de trabajo, grados de libertad, cinemática $\mathrm{y}$ programación de trayectorias) y se tienen los elementos para aplicar temas de otros cursos afines (control básico, multivariable, técnicas de optimización, estrategias no lineales, control inteligente, control secuencial, comunicaciones industriales, sistemas flexibles de manufactura, entre otros). El resultado hace parte de una etapa inicial de un laboratorio virtual-local, un ambiente de experimentación desarrollado para ser utilizado como un nodo que se enfoca en la educación remota en ingenierías.

\section{CONCLUSIONES}

Se desarrollaron dos aplicaciones de simulación, una haciendo uso del programa de National Instruments LabVIEW y otra haciendo uso del lenguaje de código abierto Java, ambas implementan el modelo cinemático, un módulo para la planificación y 
ejecución de trayectorias, un módulo para controlar remotamente los accionamientos de la etapa de potencia, otro módulo para el monitoreo de variables y un modelo 3D del Robot tipo SCARA UV-Cerma presente en el Laboratorio de Robótica Industrial de la Escuela de Ingeniería Eléctrica y Electrónica de la Universidad del Valle.

El acoplo de las interfaces con el robot real se llevó a cabo mediante la tarjeta de adquisición de datos de National Instruments NI USB-6211 y sus respectivos controladores presentes en el software LabVIEW lo cual hizo posible el mando del Robot desde la aplicación desarrollada en este software.

Se desarrolló una arquitectura cliente/servidor a través de conexiones TCP/IP donde los clientes ejecutan la aplicación Java mientras que la infraestructura del lado servidor para interactuar con el robot en el laboratorio fue creada con LabVIEW. Dado las restricciones de seguridad de la red institucional la conexión solo se puede implementar desde un cliente al interior de la misma red interna.

Cuando se desarrolló la aplicación sobre Java se encontraron ventajas debido a la fácil implementación de ecuaciones y algoritmos requeridos, pero a la hora de implementar la parte gráfica se hizo más fácil en LabVIEW debido a que este proporciona variedad de objetos destinados a la creación de interfaz humano-máquina y provee funcionalidades específicas que aceleran el desarrollo de aplicaciones que requieran la adquisición de datos, control de instrumentos y análisis de señales. La gran limitación de LabVIEW es el costo en la licencia de uso que limita el acceso, mientras que Java al ser un lenguaje de código abierto y ser multiplataforma es ideal para la creación de aplicaciones destinadas al uso por parte de una gran cantidad de usuarios.

Con este proyecto se consiguió poner en funcionamiento el Robot UV-Cerma después de varios años fuera de servicio. La plataforma es una herramienta que apoya la formación de los estudiantes de ingenie- ría en el campo de la robótica industrial, en donde se afianzan los conocimientos de cinemática directa e inversa y planificación de trayectorias, entre otros.

\section{REFERENCIAS}

[1] C. Jara, F. Candelas, and F. Torres, "Laboratorios Virtuales y Remotos Basados en EJS para la Enseñanza de Robótica Industrial," in XXVIII Jornadas de Automática, 2007.

[2] C. R. Chamorro, "Simulador Del Robot UV-Cerma," B.Sc. Thesis, Escuela de Ingeniería de Sistemas, Universidad del Valle, Cali, Colombia, 2001.

[3] C. Foix and S. Zavando, "Estándares e-Learning," Centro de Tecnologías de la Información, 2002. [Online]. Available: http://www.educ.cl/INTECEstandares_e-learning.pdf. [Accessed: 01-Oct-2013].

[4] E. Caicedo and et al, "Laboratorio Distribuido con Acceso Remoto a través de RENATA para la Experimentación en Robótica.” Universidad del Valle y Universidad del Quindío, Cali, Colombia, 2009.

[5] R. Prieto, U. Zaldíar, and R. Bernal, "Creación de un Laboratorio Virtual para Optimizar el Uso de un Laboratorio de Robótica Real," in XII Congreso Mexicano de Robótica - Comrob 2010, 2010, pp. 250255.

[6] F. Candelas, C. A. Jara, and F. Torres, "Flexible virtual and remote laboratory for teaching Robotics," in 4th Int. Conf. on Multimedia and Information \& Communication Technologies in Education, 2007, pp. 1959-1963.

[7] J. Andújar and T. Mateo, "Diseño de laboratorios virtuales y/o remotos. Un caso práctico," Rev. Iberoam. Automática e Informática Ind., vol. 7, no. 1, pp. 64-72, 2010.

[8] S. Dormido, "Control learning: present and future," Annu. Rev. Control, vol. 28, no. 1, pp. 115-136, Jan. 2004.

[9] W. Harris and D. Arnow, "Remote Shared Access To A Classroom Robotics Lab," in Resources for AI Education, AAAI 2007 Spring Symposium Series, 2007.

[10] C. A. Jara, F. A. Candelas, and F. Torres, "Virtual and remote laboratory for robotics e-learning," in 18th European Symposium on Computer Aided Process Engineering, 2008.

[11] R. Marín and P. J. Sanz, "A multimodal interface to control a robot arm via the web: a case study on remote programming," IEEE Trans. Ind. Electron., vol. 52 , no. 6 , pp. $1506-1520,2005$.

[12] M. Murtra, G. Jansà, H. Martínez, J. Domingo, J. Gámiz, A. Grau, C. C. Urgell, and E. Barcelona, "A Proposal of Remote Laboratory for Distance Training in Robotic Applications," in IEEE Conference on Emerging Technologies and Factory Automation ETFA, 2007, pp. 1180-1187.

[13] C. S. Tzafestas, N. Palaiologou, and M. Alifragis, "Virtual and Remote Robotic Laboratory: Comparative Experimental Evaluation," IEEE Trans. Educ., vol. 49, no. 3, pp. 360-369, 2006.

[14] K. Tangen, D. Groves, and J. Tomlinson, "Virtual Control of a Robotic Arm Via EMG Signals Processed Through LabView Filter Circuit," 2011. 
J. T. Buitrago-Molina et al. / Plataforma virtual para el mando local y remoto de un brazo robótico de apoyo para la educación en ingeniería

[15] I. Draganjac, V. Sesar, S. Bogdan, and Z. Kovacic, "An internet-based system for remote planning and execution of SCARA robot trajectories," in $200834 \mathrm{th}$ Annual Conference of IEEE Industrial Electronics, 2008, pp. 3485-3490.
[16] M. Alkafagee and S. Kadry, "New design of Robotics Remote lab," in International Journal of Advanced Computer Science and Applications, 2012. 